\title{
Assoziation mit Mortalität bei Dialysepflichtigkeit
}

Quelle: Ku E, Glidden DV, Johansen KL et al. Association between strict blood pressure control during chronic kidney disease and lower mortality after onset of end-stage renal disease. Kidney Int 2015; 87: 1055-1060

Thema: Es besteht eine Kontroverse, wie bei Patienten mit chronischer Niereninsuffizienz der Blutdruck kontrolliert werden soll. Randomisierte Studien konnten nicht zeigen, dass eine strikte Blutdruckkontrolle in der Lage ist, die Progression einer Nierenfunktionsstörung aufzuhalten. Prinzipiell könnte aber eine strikte Blutdruckkontrolle bei chronischer Niereninsuffizienz Vorteile für das kardiovaskuläre Risiko nach Erreichen der terminalen Niereninsuffizienz haben und dadurch potenziell das Mortalitätsrisiko positiv beeinflusst werden.

Projekt: In der MDRD ${ }^{1}$-Studie wurden 840 Patienten randomisiert mit strikter Blut- druckkontrolle (unter $92 \mathrm{mmHg}$ MAD) bzw. regulärer Blutdruckkontrolle (107 mmHg). Dies wurde zwischen 1989 und 1993 durchgeführt. Die Patienten wurden bis 2010 weiter beobachtet unter Verwendung des United States Renal Data System (USRDS) und auch dem National Death Index, um Aussagen über das Erreichen einer terminalen Niereninsuffizienz bzw. auch den Überlebensstatus zu bekommen.

Ergebnisse: Bis 2010 waren von den initial beobachteten Patienten 627 terminal niereninsuffizient geworden. Die mittlere Beobachtungsdauer betrug 19,3 Jahre. Nach Erreichen der terminalen Niereninsuffizienz gab es 142 Todesfälle in dem strikt kontrollierten Blutdruckarm und 182 Todesfälle in dem regulär kontrollierten Blutdruckarm (unadjustierte HazardRatio für die Todesfälle war 0,72 (95-\%-CI $0,58-0,89)$. In der Gesamtpopulation gab es 212 Todesfälle in dem strikten Blut- druckkontrollarm, während es 233 Todesfälle im konventionellen Blutdruckarm gab (unadjustierte Hazard-Ratio für Todesfälle war 0,82 (95-\%-CI 0,68-0,98)). Fazit: Obwohl eine strikte Blutdruckkontrolle im Verhältnis zu einer regulären Blutdruckkontrolle nicht das Erreichen einer terminalen Niereninsuffizienz verhindern kann, so hat interessanterweise auch beim Erreichen der terminalen Niereninsuffizienz eine strikte Blutdruckkontrolle in Hinblick auf die Mortalität signifikante Vorteile. Es sollte gerade bei der Blutdruckkontrolle bereits in der Prädialysephase durchaus das Langzeitüberleben der Patienten unter Dialysetherapie mit bedacht werden.

Schlüsselwörter: Blutdruckkontrolle Mortalität - Dialysepflichtigkeit

Prof. Prof. h.c. Dr. Markus van der Giet, Berlin

${ }^{1}$ Modification of Diet in Renal Disease
In den letzten Jahren haben wir erlebt, wie reihenweise ambitionierte Blutdruckziele bei der Behandlung von Patienten mit hoher Komorbidität, wie einem Diabetes, einer Niereninsuffizienz oder auch im höheren Lebensalter, durch die Leitlinien kassiert wurden. Zu Beginn des Jahrtausends war man noch optimistisch, dass durch eine differenzierte Blutdruckzieltherapie mit möglichst niedrigen Blutdruckwerten etwas Gutes für die Patienten hinsichtlich Mortalität und Morbidität erreicht wird. Allerdings zeigten die in den letzten 10 Jahren publizierten Studien, dass Patienten durch eine ambitionierte Blutdruckeinstellung nicht profitieren und möglichweise sogar Schaden erleiden können.

Nun ist die Blutdrucksenkung nicht direkt schädlich, aber Nebenwirkungen der Medikation treten häufiger auf, ohne dass der Patient hinsichtlich Mortalität und Morbidität positiv beeinflusst wird. Auch bei der Verhinderung der Progression der Niereninsuffizienz hat man viele Hoffnungen in die optimierte Behandlung des Blutdrucks gelegt. Aber bei Analyse der AASK ${ }^{1}$ und REIN-2-Studie zeigte sich, dass eine strikte Blutdruckkontrolle keinen positiven Effekt auf die verzögerte Progression einer Nie- renfunktionsstörung hat. Dies führte dazu, dass Fachgesellschaften wie die europäische Hochdruckgesellschaft oder auch die amerikanische Hypertoniegesellschaft das Blutdruckziel von unter $130 / 80 \mathrm{mmHg}$ gekippt haben und die Blutdruckziele für Patienten mit Niereninsuffizienz auf 130$140 \mathrm{mmHg}$ systolisch angehoben wurden. Die vorliegende Studie zeigt ein differenziertes und beachtenswertes Bild. Sicherlich sind die Daten durch die Analyse von Registern ggf. nicht so präzise, wie man es in einer gezielten Studie erwartet, aber die amerikanischen Register haben eine hohe Abdeckung und sind gut recherchierbar. Auch ist die Nachbeobachtungszeit von fast 20 Jahren im Durchschnitt beeindruckend bei der Größe der initialen Studie. Die Datenqualität ist auf einem hohen $\mathrm{Ni}$ veau. In der Analyse konnte gezeigt werden, dass durch strikte Blutdruckkontrolle nicht die Möglichkeit bestand, die Progression der Nierenfunktion aufzuhalten, aber die Mortalität der Patienten auch an der Dialyse wird nachhaltig durch eine gute Blutdruckkontrolle beeinflusst.

Weder die AASK noch die REIN-2-Studie haben die harten Endpunkte in der Menge gehabt, um entsprechende Aussagen ma- chen zu können. Aufgrund der Daten muss hinterfragt werden, ob die Vereinfachung der Blutdruckziele durch die Fachgesellschaften nicht etwas überambitioniert ist. Sicherlich ist die Verhinderung der Notwendigkeit zur Nierenersatztherapie bzw. der Progression der Niereninsuffizienz ein wichtiges Ziel. Wichtiger ist aber die Reduktion der Mortalität unabhängig von der Notwendigkeit der Dialyse in der Zwischenzeit. Hier scheint eine frühe strikte Blutdruckkontrolle Vorteile für die Patienten zu bringen. Da Studien mit einem Follow-up von über 20 Jahren nun schwer planbar sind, sollte versucht werden, ältere Studien ggf. nochmals zu reaktivieren und ggf. den Outcome der behandelten Patienten zu überprüfen. Sicherlich ist dies sehr ambitioniert, aber von größerer Bedeutung, um die Relevanz der Blutdruckeinstellung zu überprüfen. Gerade in den USA ist dies über die hervorragenden Register möglich.

Prof. Prof. h.c. Dr. Markus van der Giet, Berlin

'African American Study of Kidney Disease and Hypertension

${ }^{2}$ Ramipril Efficacy in Nephropathy-2 